\title{
Spin-orbit interaction in the graphitic nanocone
}

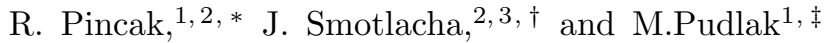 \\ ${ }^{1}$ Institute of Experimental Physics, Slovak Academy of Sciences, Watsonova 47,04353 Kosice, Slovak Republic \\ ${ }^{2}$ Bogoliubov Laboratory of Theoretical Physics, Joint Institute for Nuclear Research, 141980 Dubna, Moscow region, Russia \\ ${ }^{3}$ Faculty of Nuclear Sciences and Physical Engineering, \\ Czech Technical University, Brehova 7, 11000 Prague, Czech Republic
}

(Dated: January 18, 2021)

\begin{abstract}
The Hamiltonian for nanocones with curvature-induced spin-orbit coupling have been derived. The effect of curvature-induced spin-orbit coupling on the electronic properties of graphitic nanocones is considered. Energy spectra for different numbers of the pentagonal defects in the tip of the nanocones are calculated. It was shown that the spin-orbit interaction considerably affects the local density of states of the graphitic nanocone. This influence depends on the number of defects present at the tip of the nanocone. This property could be applied in atomic force microscopy for the construction of the probing tip.

PACS numbers: 73.22.Pr, 81.05.ue, 71.70.Ej, 72.25.-b.

Keywords: Graphitic nanocone, Spin-orbit coupling, Curvature of $\pi$ orbitals, Spins evaluated transport, Atomic force microscopy
\end{abstract}

\section{INTRODUCTION}

The spin-orbit interaction in graphene is supposed to be weak, due to the low atomic number of carbon. spinorbit coupling (SOC) in graphene has an intrinsic part, completely determined from the symmetry properties of the honeycomb lattice. The strength of this intrinsic spin-orbit coupling is rather small, due to weakness of the intraatomic spin-orbit coupling of carbon. Because of the symmetry of the honeycomb lattice this intrinsic spin-orbit coupling vanishes for hopping between neighboring atoms [1]. To get the contribution from this kind of spin-orbit coupling we have to go to the next order in the hopping. In this paper we work with the tight-binding approximation where we take into account only the hopping between nearest neighboring atoms.

In a curved graphene sheet where the symmetry of honeycomb lattice is broken there is a possibility of curvatureinduced spin-orbit coupling. A consistent approach to introduce this kind of SOC has been developed by Ando [2]. The experimental evidence for this kind of spin-orbit coupling was reported by Kuemmeth et al. [3]. It was demonstrated that in clean nanotubes the spin and orbital motion of electrons are coupled. In this work the authors measured the values of spin-orbit coupling in carbon nanotubes at various values of the magnetic field strength. It was revealed that the symmetry in electron-hole spectrum is broken. This can be caused by spin-orbit coupling.

In [4] the influence of SOC on the Kondo effect in carbon nanotube quantum dots was investigated by Fang et al. The results indicate that the spin-orbit coupling significantly changes the low-energy Kondo physics in carbon nanotube quantum dots. Recently, Steele et al. [5] have reported the large spin-orbit coupling in carbon nanotubes. It turns out that the spin-orbit coupling could be significantly enlarged by the nonzero curvature of the nanoparticle surface [6 -8]. Energy spectra and transport properties of armchair nanotubes with curvature-induced spin-orbit interactions were investigated by Pichugin et al [9]. It was reported that due to spin-orbit coupling an armchair nanotube can serve in some energy range as an spin filter. To understand clearly quantum phenomena in carbon nanoparticles the spin-orbit coupling has to be included to describe their electronic properties. The spin-orbit coupling could also be important in nanocones due to their curved surface.

In this paper, we derive the effective Hamiltonian for the graphitic nanocone with spin-orbit coupling induced by curvature. The structure of the paper is as follows. In Sec. II, we introduce an explicit formula for the eigenspectrum of the Hamiltonian with full curvature-induced spin-orbit coupling in a carbon nanocone. The solution is derived in the Appendices. In Sec. III, we present numerical results for all of our calculations. The main conclusions are summarized in Sec. IV.

\footnotetext{
*Electronic address: pincak@saske.sk

${ }^{\dagger}$ Electronic address: smota@centrum.cz

‡Electronic address: pudlak@saske.sk
} 


\section{DIRAC EQUATION FOR CURVATURE-INDUCED SPIN-ORBIT COUPLING}

The Hamiltonian has been derived following the method of Ando [2]. Adapting the derivation of Hamiltonian in [10], we introduce the curvature-induced spin-orbit interaction on the nanocone. We start with the Hamiltonian for the nanoconical surface without the spin-orbit coupling and pseudopotential [10, 11]. Due to the rotational symmetry of the nanocone, we choose the radial and angular coordinates $r, \varphi$. Here, we will often use the coordinate $R$. It is the distance between the point $\mathbf{r}$ on the surface and the intersection of the conical axis with the line perpendicular to surface at point $\mathbf{r}$ (Fig. 1). It satisfies $R=\frac{(1-\eta) r}{\sqrt{\eta(2-\eta)}}$, where $\eta=N_{d} / 6$ is the Frank index depending on $N_{d}$, the number of defects in the nanoconical tip.

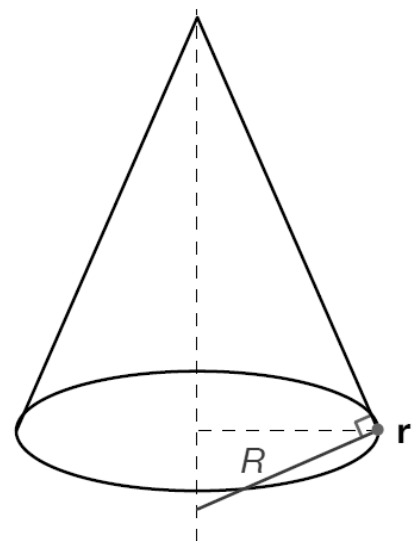

FIG. 1: The notation of the distances in the nanocone.

The Hamiltonian has the form

$$
\hat{H}=\left(\begin{array}{cc}
H_{1} & 0 \\
0 & H_{-1}
\end{array}\right) \quad H_{s}=\mathrm{i} \hbar v\left\{\tau^{y} \partial_{r}-\tau^{x} r^{-1}\left[(1-\eta)^{-1}\left(s \partial_{\varphi}-\frac{3}{2} \mathrm{i} \eta\right)-\frac{1}{2} \tau^{z}\right]\right\},
$$

where $\tau^{x}, \tau^{y}, \tau^{z}$ are the Pauli matrices, $s= \pm 1$ denotes the value of the $K$ spin.

Now, we will supply the terms corresponding to the spin-orbit interaction. It means that we perform the substitutions

$$
\partial_{r} \rightarrow \partial_{r}-\frac{\delta \gamma^{\prime}}{4 \gamma} b_{\varphi}^{\varphi} \sigma_{x}(\vec{r}), \quad \mathrm{i} \partial_{\varphi} \rightarrow \mathrm{i} \partial_{\varphi}+s(1-\eta) A_{y} \sigma_{y}
$$

Here, $\sigma^{x}, \sigma^{y}, \sigma^{z}$ are the Pauli matrices corresponding to spin of electrons and we use the linear combination $\sigma_{x}(\vec{r})=$ $\sigma^{x} \cos \varphi-\sigma^{z} \sin \varphi$. The curvature of the surface is included in the curvature tensor $b_{\varphi}^{\varphi}=\frac{1}{R}$ and in the parameter $A_{y}=s \frac{2 \delta p}{(1-\eta)} \sqrt{\eta(2-\eta)}$, which depends on the Frank index. The other parameters are described in [2] and they have the following meaning: the hopping integrals $\gamma=-\sqrt{3} V_{p p}^{\pi} a / 2, \gamma^{\prime}=\sqrt{3}\left(V_{p p}^{\sigma}-V_{p p}^{\pi}\right) a / 2$, where $a$ is the length of the primitive translation vector $\left(a=\sqrt{3} d \simeq 2.46 \AA . \AA\right.$ is the distance between atoms in the unit cell); $V_{p p}^{\sigma}$ and $V_{p p}^{\pi}$ are the transfer integrals for the $\sigma$ and $\pi$ orbitals, respectively, in flat 2D graphite. Next, $p=1-3 \gamma^{\prime} / 8 \gamma, \delta=\frac{1}{3} \frac{\Delta}{\epsilon_{\pi \sigma}}$, where

$$
\Delta=i \frac{3 \hbar}{4 m^{2} c^{2}}\left\langle x\left|\frac{\partial V}{\partial x} p_{y}-\frac{\partial V}{\partial y} p_{x}\right| y\right\rangle
$$

( $V$ is the atomic potential) and $\epsilon_{\pi \sigma}=\epsilon_{2 p}^{\pi}-\epsilon_{2 p}^{\sigma}$. The energy $\epsilon_{2 p}^{\sigma}$ is the energy of $\sigma$-orbitals that are localized between carbon atoms. The energy $\epsilon_{2 p}^{\pi}$ is the energy of $\pi$-orbitals that are directed perpendicular to the nanotubular surface. The following values of the parameters are chosen: $\delta$ is of the order between $10^{-3}$ and $10^{-2}, \frac{\gamma^{\prime}}{\gamma} \sim \frac{8}{3}, p \sim 0.1, V_{p p}^{\pi} \approx-3$ $\mathrm{eV}$ and $V_{p p}^{\sigma} \approx 5 \mathrm{eV}, p \approx 0.1$ and $\delta \approx 0.01[2]$. Now, we will do the transformation

$$
\hat{H}^{\prime}=e^{\mathrm{i} \frac{\sigma_{y}}{2} \varphi} \hat{H} e^{-\mathrm{i} \frac{\sigma_{y}}{2} \varphi}
$$


With the aid of this transformation we describe the motion of an electron in the local coordinate frame which moves with the electron on the nanocone surface. Now we get

$$
\hat{H}^{\prime}=\hbar v\left(\begin{array}{cc}
0 & \partial_{r}-\mathrm{i} s \frac{1}{r(1-\eta)} \partial_{\varphi}-\frac{1}{2 r}-\mathrm{i} \frac{1}{r} \xi_{x} \sigma_{x}-\frac{3}{2} \frac{\eta}{(1-\eta) r}-\frac{\xi_{y} \sigma_{y}}{r} \\
0 & 0
\end{array}\right)
$$

where the parameters $\xi_{x}, \xi_{y}$ describe the strength of the spin-orbit interaction:

$$
\xi_{x}=\frac{\delta \gamma^{\prime} \sqrt{\eta(2-\eta)}}{4(1-\eta) \gamma}, \quad \xi_{y}=A_{y}+\frac{1}{2(1-\eta)}
$$

Now, we are solving the equation

$$
\hat{H}^{\prime} \psi(r, \varphi)=E \psi(r, \varphi)
$$

where, considering the rotational symmetry of the solution, we do the factorization

$$
\psi(r, \varphi)=e^{\mathrm{i} j \varphi}\left(\begin{array}{c}
f_{j \uparrow}(r) \\
f_{j \downarrow}(r) \\
g_{j \uparrow}(r) \\
g_{j \downarrow}(r)
\end{array}\right)
$$

Then, after the substitution of this expression into (7) and performing the differentiations with respect to $\varphi$, we have

$$
\left(\begin{array}{cccc}
0 & 0 & \partial_{r}+\frac{F}{r} & -\frac{\mathrm{i}}{r} C \\
0 & 0 & -\frac{\mathrm{i}}{r} D & \partial_{r}+\frac{F}{r} \\
-\partial_{r}+\frac{F-1}{r} & \frac{\mathrm{i}}{r} D & 0 & 0 \\
\frac{\mathrm{i}}{r} C & -\partial_{r}+\frac{F-1}{r} & 0 & 0
\end{array}\right)\left(\begin{array}{c}
f_{j \uparrow}(r) \\
f_{j \downarrow}(r) \\
g_{j \uparrow}(r) \\
g_{j \downarrow}(r)
\end{array}\right)=E\left(\begin{array}{c}
f_{j \uparrow}(r) \\
f_{j \downarrow}(r) \\
g_{j \uparrow}(r) \\
g_{j \downarrow}(r)
\end{array}\right)
$$

where

$$
F=\frac{s j}{1-\eta}-\frac{3}{2} \frac{\eta}{1-\eta}+\frac{1}{2}, \quad C=\xi_{x}-\xi_{y}, \quad D=\xi_{x}+\xi_{y} .
$$

The parameter $s= \pm 1$. The solution of (9) is given in the Appendix.

\section{ELECTRONIC PROPERTIES OF THE CONICAL NANOSTRUCTURE}

For $\beta=1$ and $E=1$, we see the solution of (9) in Fig. 2. The signs $\uparrow, \downarrow$ in the indices are replaced here by the signs ,+- , respectively. The graphs for $\operatorname{Re} f_{\downarrow}, \operatorname{Re} g_{\downarrow}, \operatorname{Im} f_{\uparrow}$ and $\operatorname{Im} g_{\uparrow}$ are missing - they provide the zero solution. We can see that for the cases of 1 and 2 defects, the modules of $f_{\downarrow}, f_{\uparrow}$ and $g_{\downarrow}, g_{\uparrow}$, respectively, coincide - the existing effect of the spin-orbit interaction is still not strong enough to split the appropriate components of the wave-function.
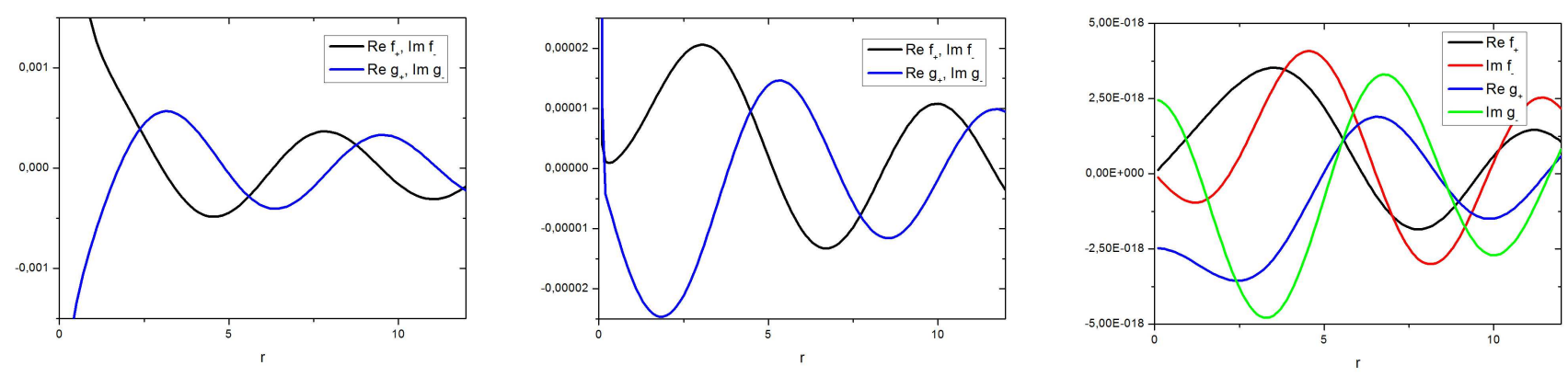

FIG. 2: Solution of the system (9) for the case of $N_{d}=1$ (left), $N_{d}=2$ (middle), $N_{d}=3$ (right) and $E=1$. 
This solution has a form similar to Bessel functions of the first or the second kind $\left(J_{j}\right.$ or $\left.Y_{j}\right)$. This correspondence is derived in Appendix B for the case of $N_{d}=1$ defect and $j=2$. For other values of the energies or the parameter $j$, this occurrence will be similar.

For the normalized case, given energy and value of $j$, the local density of states $(L D o S)$ is defined as

$$
L D o S(E, r)=\left|f_{j, E \uparrow}(r)\right|^{2}+\left|f_{j, E \downarrow}(r)\right|^{2}+\left|g_{j, E \uparrow}(r)\right|^{2}+\left|g_{j, E \downarrow}(r)\right|^{2} .
$$
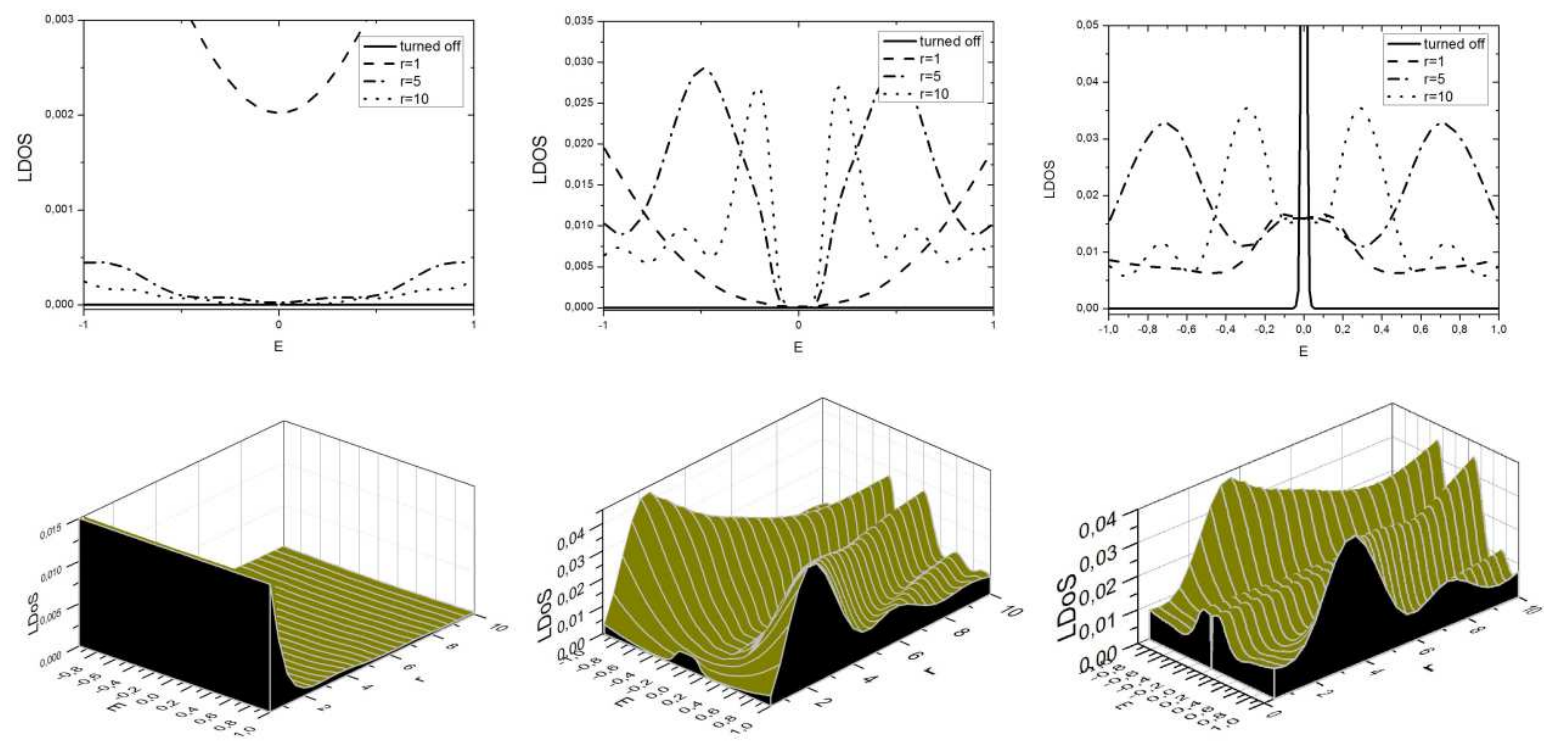

FIG. 3: 2D and 3D (bottom) graphs of the local density of states with and without (turned off) spin-orbital interaction for different distances $r$ from the tip. $N_{d}=1$ (left), $N_{d}=2$ (middle) and $N_{d}=3$ (right).

The numerical results are depicted in Fig. 3. The solution without the spin-orbit interaction is the turned off case. For this case, the constants $C$ and $D$ would be equal to zero and the system (9) would be a 4-dimensional analogy of the 2-dimensional case without the spin-orbit interaction (described by Pincak et al. in [11]) and with the exclusion of the effect of the pseudopotential. This evidence can also be derived from the above plots in Fig. 2, where the modules of the first and second or the third and fourth component, respectively, are not split unless the number of the defects in the conical tip exceeds 2 .

\section{CONCLUSION}

An effective mass Hamiltonian was derived for a carbon nanocone in the presence of curvature-induced spin-orbit interaction. Within our approach, we solved analytically the eigenvalue problem for the effective mass Hamiltonian for electrons on the curved surface with the spin-orbit interaction. In particular, we obtained explicit expressions for a low-energy spectrum and eigenstates of carbon nanocones. The $L D o S$ in the graphitic nanocone near the tip in the case of spin-orbit interactions were computed numerically. These findings have been used to analyze electronic properties of carbon nanocones with curvature-induced SOC at different limits. We see that the spinorbit interaction considerably affects the local density of states of the graphic nanocone; the higher the number of defects, the bigger the effect. One of the reason is that the more defects present near the tip, the bigger the curvature of the nanocone in the vicinity of the tip, and so also the bigger the effect of the imposed spin-orbit interaction. The localization of the electrons as shown in Fig. 3 makes the graphitic nanocone a possible candidate for the construction of a new type of scanning probe in atomic force microscopy [12 15].

ACKNOWLEDGEMENTS - The work was supported by the Science and Technology Assistance Agency under Contract No. APVV-0171-10, VEGA Grant No. 2/0037/13 and Ministry of Education Agency for Structural Funds of EU in frame of project 26220120021, 26220120033 and 26110230061. R. Pincak would like to thank the TH division in CERN for hospitality. 


\section{Appendix A: Solution of the Dirac equation}

We want to find the solution of (9) in the form

$$
\begin{aligned}
f_{j \uparrow}(r)=e^{\frac{\alpha}{r}+\beta r} \sum_{k=0}^{\infty} a_{k} r^{\xi+k}, & f_{j \downarrow}(r)=e^{\frac{\alpha}{r}+\beta r} \sum_{k=0}^{\infty} b_{k} r^{\xi+k}, \\
g_{j \uparrow}(r)=e^{\frac{\alpha}{r}+\beta r} \sum_{k=0}^{\infty} c_{k} r^{\xi_{1}+k}, & g_{j \downarrow}(r)=e^{\frac{\alpha}{r}+\beta r} \sum_{k=0}^{\infty} d_{k} r^{\xi_{1}+k} .
\end{aligned}
$$

After the substitution, we get $\xi=\xi_{1}-2$ and

$$
\begin{aligned}
& -\alpha c_{0}=E a_{0}, \quad-\alpha c_{1}+\xi_{1} c_{0}+F c_{0}-\mathrm{i} C d_{0}=E a_{1}, \quad-\alpha d_{0}=E b_{0}, \quad-\alpha d_{1}+\xi_{1} d_{0}+F d_{0}-\mathrm{i} D c_{0}=E b_{1}, \\
& \alpha a_{0}=0, \quad \alpha a_{1}+\xi a_{0}+(F-1) a_{0}+\mathrm{i} D b_{0}=0, \quad \alpha b_{0}=0, \quad \alpha b_{1}-\xi b_{0}+(F-1) b_{0}+\mathrm{i} C a_{0}=0, \\
& \alpha a_{2}-\beta a_{0}+(F-\xi-2) a_{1}+\mathrm{i} D b_{1}=0, \quad \alpha b_{2}-\beta b_{0}+(F-\xi-2) b_{1}+\mathrm{i} C a_{1}=0, \\
& \alpha a_{3}-\beta a_{1}+(F-\xi-3) a_{2}+\mathrm{i} D b_{2}=0, \quad \alpha b_{3}-\beta b_{1}+(F-\xi-3) b_{2}+\mathrm{i} C a_{2}=0 .
\end{aligned}
$$

For the other indices, we get the system of recurrence equations

$$
\begin{gathered}
-\alpha c_{k}+\beta c_{k-2}+\left(F+\xi_{1}+k-1\right) c_{k-1}-\mathrm{i} C d_{k-1}=E a_{k}, \quad-\alpha d_{k}+\beta d_{k-2}+\left(F+\xi_{1}+k-1\right) d_{k-1}-\mathrm{i} D c_{k-1}=E b_{k}, \quad(\mathrm{~A} 7) \\
-\alpha a_{k}+\beta a_{k-2}-\left(F-\xi_{1}+2-k\right) a_{k-1}-\mathrm{i} D b_{k-1}=-E c_{k-4}, \quad-\alpha b_{k}+\beta b_{k-2}-\left(F-\xi_{1}+2-k\right) b_{k-1}-\mathrm{i} C a_{k-1}=-E d_{k-4} .
\end{gathered}
$$

If we suppose that $\alpha \neq 0$, we get the zero solution. So for the nontrivial solution $\alpha=0$ and as follows from the first and the third equation in (A3), in this case the coefficients $a_{0}$ and $b_{0}$ must be also zero. Then, from the system

$$
\left(F-\xi_{1}\right) a_{1}+\mathrm{i} D b_{1}=0, \quad\left(F-\xi_{1}\right) b_{1}+\mathrm{i} C a_{1}=0
$$

follows:

$$
\xi_{1}=F \pm \mathrm{i} \sqrt{C D}, \quad b_{1}= \pm \sqrt{\frac{C}{D}} a_{1}
$$

From the system

$$
\left(F+\xi_{1}\right) c_{0}-\mathrm{i} C d_{0}=E a_{1}, \quad\left(F+\xi_{1}\right) d_{0}-\mathrm{i} D c_{0}=E b_{1},
$$

we get

$$
c_{0}=\frac{\left(F+\xi_{1}\right) a_{1}+\mathrm{i} C b_{1}}{\left(F+\xi_{1}\right)^{2}+C D} E, \quad d_{0}=\frac{E b_{1}+\mathrm{i} D c_{0}}{F+\xi_{1}} .
$$

And from the system

$$
\left(F-\xi_{1}-1\right) a_{2}+\mathrm{i} D b_{2}=\beta a_{1}, \quad\left(F-\xi_{1}-1\right) b_{2}+\mathrm{i} C a_{2}=\beta b_{1},
$$

follows

$$
b_{2}=\beta \frac{\left(F-\xi_{1}-1\right) b_{1}-\mathrm{i} C a_{1}}{\left(F-\xi_{1}-1\right)^{2}+C D}, \quad a_{2}=\frac{\beta a_{1}-\mathrm{i} D b_{2}}{F-\xi_{1}-1} .
$$


Here, $\beta$ is a free parameter. The following coefficients we get from the recurrence equations.

Now excluding the functions $f_{j \uparrow}, f_{j \downarrow}$ with the help of the first two equations in (9), we get a simplified system

$$
\begin{aligned}
& -r^{2} g_{j \uparrow}^{\prime \prime}(r)+(2 F-1) r g_{j \uparrow}^{\prime}(r)+\left[F(F-2)+D^{2}\right] g_{j \uparrow}(r)+\mathrm{i} r(C+D) g_{j \downarrow}^{\prime}(r)-\mathrm{i}(C-D) F g_{j \downarrow}(r)=E^{2} r^{2} g_{j \uparrow}(r), \\
& \operatorname{i} r(C+D) g_{j \uparrow}^{\prime}(r)+\mathrm{i}(C F-D) g_{j \uparrow}(r)-\mathrm{i} r(F-1) D g_{j \uparrow}(r)-r^{2} g_{j \downarrow}^{\prime \prime}(r)-r g_{j \downarrow}^{\prime}(r)+\left(C^{2}+F^{2}\right) g_{j \downarrow}(r)=E^{2} r^{2} g_{j \downarrow}(r) .
\end{aligned}
$$

To solve the problem an iteration method is used. For this purpose, we divide the components of the solution into the real and the imaginary part:

$$
g_{j \uparrow}=\operatorname{Re} g_{j \uparrow}+\mathrm{i} \operatorname{Im} g_{j \uparrow}, \quad g_{j \downarrow}=\operatorname{Re} g_{j \downarrow}+\mathrm{i} \operatorname{Im} g_{j \downarrow} .
$$

Now, for a given $j$, we denote

$$
\operatorname{Re} g_{j \uparrow}=G_{1}, \quad \operatorname{Im} g_{j \downarrow}=G_{2}
$$

and if we suppose that the conditions

$$
\operatorname{Im} f_{j \uparrow}=\operatorname{Re} f_{j \downarrow}=\operatorname{Im} g_{j \uparrow}=\operatorname{Re} g_{j \downarrow}=0 .
$$

are satisfied for the analytical solution, for the nonzero components of $g_{j \uparrow}, g_{j \downarrow}$ we have

$$
\begin{gathered}
-r^{2} G_{1}^{\prime \prime}(r)+(2 F-1) r G_{1}^{\prime}(r)+\left[F(F-2)+D^{2}\right] G_{1}(r)-r(C+D) G_{2}^{\prime}(r)+(C-D) F G_{2}(r)=E^{2} r^{2} G_{1}(r), \\
r(C+D) G_{1}^{\prime}(r)+(C F-D) G_{1}(r)-r(F-1) D G_{1}(r)-r^{2} G_{2}^{\prime \prime}(r)-r G_{2}^{\prime}(r)+\left(C^{2}+F^{2}\right) G_{2}(r)=E^{2} r^{2} G_{2}(r) .
\end{gathered}
$$

This system can be rewritten into the form

$$
\begin{gathered}
r^{2} G_{1}^{\prime \prime}(r)-(2 F-1) r G_{1}^{\prime}(r)+\left[E^{2} r^{2}-F(F-2)-D^{2}\right] G_{1}(r)=\mathfrak{D}_{1}\left(G_{2}(r)\right), \\
r^{2} G_{2}^{\prime \prime}(r)+r G_{2}^{\prime}(r)+\left[E^{2} r^{2}-C^{2}-F^{2}\right] G_{2}(r)=\mathfrak{D}_{2}\left(G_{1}(r)\right),
\end{gathered}
$$

where $\mathfrak{D}_{1}, \mathfrak{D}_{2}$ on the right-hand side denote the differential operators. If we exclude them, we get the homogeneous parts of the system. For the second equation it gives the Bessel equation, for the first equation it gives a Bessel-like equation. It is not a problem to find a solution for this homogeneous system. We can try to find a particular solution for the inhomogeneous system in this way: let $G_{1-}^{(0)}, G_{1+}^{(0)}$ and $G_{2-}^{(0)}, G_{2+}^{(0)}$, respectively, denote the solution for the homogeneous system (actually, $G_{2-}^{(0)}, G_{2+}^{(0)}$ are the Bessel functions), then the particular solutions could be searched with the help of the method of variation of the constants, i.e. in the form

$$
G_{1}(r)=C_{1-}(r) G_{1-}^{(0)}(r)+C_{1+}(r) G_{1+}^{(0)}(r), \quad G_{2}(r)=C_{2-}(r) G_{2-}^{(0)}(r)+C_{2+}(r) G_{2+}^{(0)}(r) .
$$

Of course, there is the question of in which form the functions $G_{1}(r), G_{2}(r)$ would be substituted into the arguments of the operators $\mathfrak{D}_{1}, \mathfrak{D}_{2}$. In the first step, we could make a statement $C_{2-}(r)=C_{2+}(r)=1$ and substitute $G_{2}(r)$ into the right-hand side of (A22). In this way, we get the form of $C_{1-}(r), C_{1+}(r)$ and we can substitute $G_{1}(r)$ into the right-hand side of (A23) acquiring more precise values of $C_{2-}(r), C_{2+}(r)$. This procedure can be repeated unless we achieve the required precision. We make a suggestion that the solution of (9) has a form of the Bessel-like functions. This estimate will be verified in the next section by comparison of the coefficients $a_{n}, b_{n}, c_{n}, d_{n}$ with the coefficients corresponding to the Taylor series of the Bessel functions. 


\section{Appendix B: Verification of the similarity between the solution and Bessel functions}

Now we want to prove the correspondence between the solution of (9) and the Bessel functions. But first, we need to change the scale of the corresponding real or imaginary part of $f_{j \uparrow, \downarrow}$ or $g_{j \uparrow, \downarrow}$ such that the null points of the given function and the corresponding Bessel function correlate (see Fig. 4). Then, we will do the described comparison for the case of 1 defect and $j=2$ and the corresponding Bessel functions $J_{1}$ and $J_{2}$ (see Table \). The re-scaling we do numerically. For the value $E=0.75$ and the unnormalized case, the re-scaled form of the solution of (9) has the form

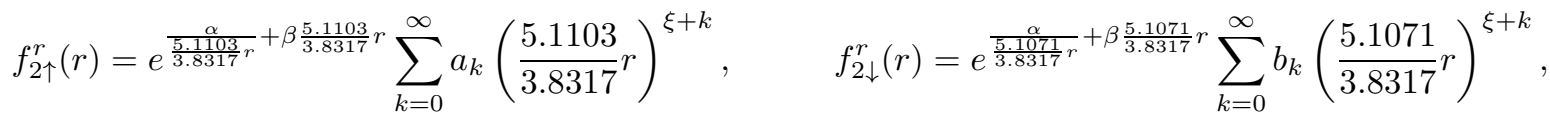

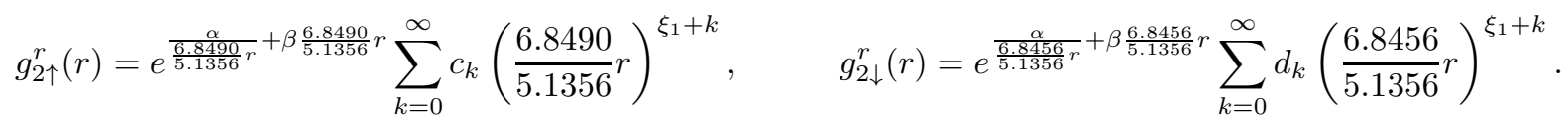

We can do a shortcut of these expressions into the first 10 members of the expansion. Here, we choose $\alpha=0$ and $\beta=1$. Recalling that for the given values of the parameters, $\xi_{1}=1.99987 \doteq 2$, we have

$$
\begin{gathered}
\operatorname{Re} f_{2 \uparrow}^{r}(r)=f_{2 \uparrow}^{r}(r) \doteq 1.33 r-0.17 r^{3}+6.95 \cdot 10^{-3} r^{5}-1.45 \cdot 10^{-4} r^{7}+1.81 \cdot 10^{-6} r^{9}+\mathcal{O}\left(r^{10}\right), \\
\operatorname{Im} f_{2 \downarrow}^{r}(r)=f_{2 \downarrow}^{r}(r) \doteq-1.33182 r+0.17 r^{3}-6.93 \cdot 10^{-3} r^{5}+1.44 \cdot 10^{-4} r^{7}-1.80 \cdot 10^{-6} r^{9}+\mathcal{O}\left(r^{10}\right), \\
\operatorname{Re} g_{2 \uparrow}^{r}(r)=g_{2 \uparrow}^{r}(r) \doteq 0.33 r^{2}-2.78 \cdot 10^{-2} r^{4}+8.69 \cdot 10^{-4} r^{6}-1.45 \cdot 10^{-5} r^{8}+1.51 \cdot 10^{-7} r^{10}+\mathcal{O}\left(r^{11}\right), \\
\operatorname{Im} g_{2 \downarrow}^{r}(r)=g_{2 \downarrow}^{r}(r) \doteq-0.33 r^{2}+2.77 \cdot 10^{-2} r^{4}-8.67 \cdot 10^{-4} r^{6}+1.45 \cdot 10^{-5} r^{8}-1.50 \cdot 10^{-7} r^{1} 0+\mathcal{O}\left(r^{11}\right) .
\end{gathered}
$$

The corresponding expansions for the Bessel functions $J_{1}, J_{2}$ have the form

$$
\begin{gathered}
J_{1}(r) \doteq 0.5 r-0.06 r^{3}+2.60 \cdot 10^{-3} r^{5}-5.43 \cdot 10^{-5} r^{7}+6.78 \cdot 10^{-7} r^{9}+\mathcal{O}\left(r^{10}\right), \\
J_{2}(r) \doteq 0.125 r^{2}-0.01 r^{4}+3.26 \cdot 10^{-4} r^{6}-5.43 \cdot 10^{-6} r^{8}+5.65 \cdot 10^{-8} r^{1} 0+\mathcal{O}\left(r^{11}\right) .
\end{gathered}
$$

Now, if we make a comparison of the coefficients corresponding to the power series of $\operatorname{Re} f_{2 \uparrow}^{r}, \operatorname{Im} f_{2 \downarrow}^{r}$, respectively, with the coefficients corresponding to $J_{1}$ and of the coefficients corresponding to the power series of $\operatorname{Re} g_{2 \uparrow}^{r}, \operatorname{Im} g_{2 \downarrow}^{r}$, respectively, with the coefficients corresponding to $J_{2}$, we find that more or less, for a concrete pair of functions, the ratio of the coefficients remains constant and it approaches these values:

$$
\frac{\operatorname{Re} f_{2 \uparrow}^{r}(r)}{J_{1}(r)} \doteq 2.67, \quad \frac{\operatorname{Im} f_{2 \downarrow}^{r}(r)}{J_{1}(r)} \doteq-2.66, \quad \frac{\operatorname{Re} g_{2 \uparrow}^{r}(r)}{J_{2}(r)} \doteq 2.67, \quad \frac{\operatorname{Im} g_{2 \downarrow}^{r}(r)}{J_{2}(r)} \doteq-2.66
$$

In Table I we see the concrete forms of the Bessel functions which correspond to the case $N_{d}=1$ defect and different values of the parameter $j$. However, the higher the value of $j$ is, the more spread this correspondence is. On the whole, we can say that for the case of $N_{d}=1$ defect and values of $j \geq 2$, the analytical expression for the solution can be approximated as

$$
\operatorname{Re} f_{j \uparrow} \sim J_{j-1}(E r), \quad \operatorname{Im} f_{j \downarrow} \sim J_{j-1}(E r), \quad \operatorname{Re} g_{j \uparrow} \sim J_{j}(E r), \quad \operatorname{Im} g_{j \downarrow} \sim J_{j}(E r) .
$$

For the case of $N_{d}=2$ and $N_{d}=3$ defects, the corresponding tables would be more complicated, and the solution would be a linear combination of Bessel functions of different kinds. 


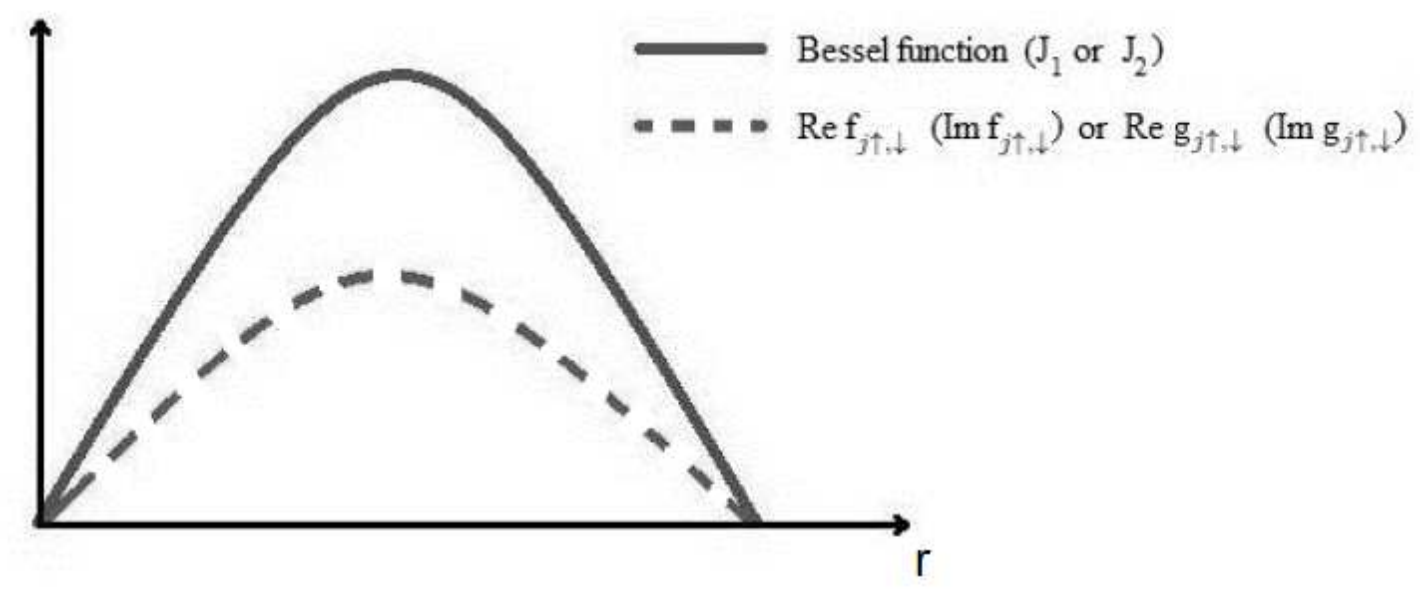

FIG. 4: Comparison of the real or imaginary part of $f_{j \uparrow, \downarrow}\left(g_{j \uparrow, \downarrow}\right)$ and of the corresponding Bessel function. Here, the $r$ coordinate denotes the distance from a tip. We have a boundary of a nanocone at $r_{0}=20$. We have zero probability of finding an electron at a boundary.

TABLE I: Correspondence of the Bessel function with solutions of (9) for $N_{d}=1$ defect and different values of $j$.

\begin{tabular}{ccccc}
\hline$j$ & $\operatorname{Re} f_{j \uparrow}$ & $\operatorname{Im} f_{j \downarrow}$ & $\operatorname{Re} g_{j \uparrow}$ & $\operatorname{Im} g_{j \downarrow}$ \\
\hline 0 & $-Y_{2}$ & $-Y_{2}$ & $Y_{1}$ & $Y_{1}$ \\
1 & $-Y_{1}$ & $-Y_{1}$ & $J_{1}$ & $J_{1}$ \\
2 & $J_{1}$ & $J_{1}$ & $J_{2}$ & $J_{2}$ \\
3 & $J_{2}$ & $J_{2}$ & $J_{3}$ & $J_{3}$ \\
4 & $J_{3}$ & $J_{3}$ & $J_{4}$ & $J_{4}$ \\
\hline
\end{tabular}

[1] C. L. Kane and E. J. Mele, Phys. Rev. Lett. 95 (2005) 226801.

[2] T. Ando, J. Phys. Soc. Jpn. 69 (2000) 1757.

[3] F. Kuemmeth, S. Ilani, D. C. Ralph, P. L. McEuen, Nature 452 (2008) 448.

[4] T. Fang, W. Zuo and H. Luo, Phys. Rev. Lett. 101 (2008) 246805.

[5] G. A. Steele et al., Nature Communications DOI:10.1038/ncomms2584.

[6] D. Huertas-Hernando, F. Guinea and A. Brataas, Phys. Rev. B 74 (2006) 155426.

[7] J. S. Jeong, J. Shin and H. W. Lee, Phys. Rev. B 84 (2011) 195457.

[8] M. del Valle, M. Marganska and M. Grifoni, Phys. Rev. B 84 (2011) 165427.

[9] K. N. Pichugin, M.Pudlak and R.G. Nazmitdinov, Eur. Phys. J. B 87 (2014) 124.

[10] Yu. A. Sitenko, N. D. Vlasii, Nucl. Phys. B 787 (2007) 241.

[11] R. Pincak, J. Smotlacha and M. Pudlak, Physica B 441 (2014) 58.

[12] I.-C. Chen, L.-H. Chen et al., Appl. Phys. Lett. 88 (2006) 153102.

[13] J. Sripirom, S. Noor, U. Kohler, A. Schulte, Carbon 49 (2011) 2402-2412.

[14] Ö. Karc, M. Dede, and A. Oral, Review of Scientific Instruments 85 (2014) 103705 .

[15] T. Nishio, Q. Chen, W. Gillijns et al., Phys. Rev. B 77 (2008) 012502. 